\title{
82.
}

\section{ON THE TRIADIC ARRANGEMENTS OF SEVEN AND FIFTEEN THINGS.}

[From the Philosophical Magazine, vol. xxxvII. (1850), pp. 50-53.]

THERE is no difficulty in forming with seven letters, $a, b, c, d, e, f, g$, a system of seven triads containing every possible duad; or, in other words, such that no two triads of the system contain the same duad. One such system, for instance, is

$$
a b c, \quad a d e, \quad a f g, \quad b d f, \quad b e g, \quad c d g, \quad c e f ;
$$

and this is obviously one of six different systems obtained by permuting the letters $a, b, c$. We have therefore six different systems containing the triad $a b c$; and there being the same number of systems containing the triads $a b d$, abe, $a b f$ and $a b g$ respectively, there are in all thirty-five different systems, each of them containing every possible duad. It is deserving of notice, that it is impossible to arrange the thirty-five triads formed with the seven letters into five systems, each of them possessing the property in question. In fact, if this could be done, the system just given might be taken for one of the systems of seven triads. With this system we might (of the systems of seven triads which contain the triad $a b d$ ) combine either the system $a b d, \quad a c g, a e f, \quad b c e, \quad b f g, \quad d c f, \quad d e g$,

or the system

$$
a b d, \quad a c f, \quad a e g, \quad b c g, \quad b e f, \quad d c e, \quad d f g ;
$$

(but any one of the other abd systems would be found to contain a triad in common with the given $a b c$ system, and therefore cannot be made use of: for instance, the system $a b d, a c g, a e f, b c f, b e g, d c e, d f g$ contains the triad beg in common with the given $a b c$ system): and whichever of the two proper $a b d$ systems we select to combine with the given $a b c$ system, it will be found that there is no abe system which does not contain some triad in common, either with the $a b c$ system or with the abd system.

c. 
The order of the letters in a triad has been thus far disregarded. There are some properties which depend upon considering the triads obtained by cyclical permutations of the three letters as identical, but distinct from the triads obtained by a permutation of two letters, or inversion. Thus $a b c, b c a, c a b$ are to be considered as identical inter $s e$, but distinct from the triads $a c b, c b a, b a c$, which are also identical inter se. I write down the system (equivalent, as far as the mere combination of the letters is concerned, to the system at the commencement of this paper)

$$
a d e, \quad a f g, \quad b d f, \quad b g e, \quad c d g, \quad c e f, \quad c b a,
$$

derived, it is to be observed, from a pair of triads, ade, afg, by a cyclical permutation of the $e, f, g$, and by successively changing the $a$ into $b$ and into $c$, the remaining triad of the system being the letters $a, b, c$ taken in an inverse order. Let it be proposed to derive the system in the same manner from any other two triads of the system; for instance, from the triads $a c b$, ade. The process of derivation gives

$$
a c b, \text { ade, } g c d, \quad g e b, f c e, f b d, f g a,\left({ }^{1}\right)
$$

which is, in fact, the original system. But attempt to derive the system from the two triads $e b g$, efc, the process of derivation gives

$$
e b g, \quad e f c, \quad d b f, \quad d c g, \quad a b c, \quad a g f, \quad a d e,
$$

which is not the original system, inasmuch as the triads $d b f, d c g, a b c, a g f$ are inversions of the triads $b d f, c d g, c b a, a f g$ of the original system. The point to be attended to, however, is, that both triads of the pair $d b f$, $d c g$, or of the pair $a b c$, agf, are inversions of the triads of the corresponding pair in the original system; the pair is either reproduced (as the pair $e f c$, $d b f$ ), or there is an inversion of both triads. Where there is no such inversion of the triads of a pair, the system may be said to be properly reproduced; and where there is inversion of the triads of one or more pairs, to be improperly reproduced. There is no difficulty in seeing that the system is properly reproduced from a pair of triads containing in common any one of the letters $a, b, c$ or $d$, and improperly reproduced from pairs of triads containing in common any one of the letters $d$, $e$ or $f$. It is owing to the reproduction, proper or improper, of the system from any pair of duads that it is possible to form a system of "octaves" analogous to the quaternions of Sir William Hamilton; the impossibility of a corresponding system of fifteen imaginary quantities arises from the circumstance of there being always, in whatever manner the system of triads is formed, an inversion of a single triad of some one or more pairs of triads containing a letter in common. When the system is considered as successively derived from different pairs, the system is not according to the previous definition reproduced either properly or improperly. A system of triads having the necessary properties with respect to the mere combination of the letters (viz. that $\alpha \beta \gamma$ and $\alpha \delta \epsilon$ being any two

1 The order of the letters $f, g$ is selected so as to reproduce the original system so far as the mere combination of the letters is concerned. 
triads having a letter in common, there shall be triads such as $\zeta \beta \delta, \zeta \gamma \epsilon$, and $\eta \beta \epsilon$, $\eta \gamma \delta$ ) may easily be found; the system to be presently given of the triads of fifteen things would answer the purpose. And so would many other systems.

Dropping the consideration of the order of the letters which form a triad, I pass to the case of a system of fifteen letters, $a, b, c, d, e, f, g, h, i, j, k, l, m, n, o$. It is possible in this case, not only to form systems of thirty-five triads containing every possible duad, but this can be done in such manner that the system of thirtyfive triads can be arranged in seven systems of five triads, each of these systems containing the fifteen letters ${ }^{1}$. My solution is obtained by a process of derivation from the arrangements $a b . c f . d g . e h$ and $a b . c d . e f . g h$ as follows; viz. the triads are

$\begin{array}{lllllll}i a b & j a c & k a f & \text { lad } & \text { mag } & \text { nae } & \text { oah } \\ i c f & j f b & k b c & \text { lce } & \text { mch } & \text { ncd } & \text { ocg } \\ i d g & j d e & k d h & l g b & \text { mbd } & n g f & \text { ofd } \\ i e h & j h g & k g e & \text { lhf } & \text { mfe } & \text { nhb } & \text { obe }\end{array}$

and a system formed with $i, j, k, l, m, n o$, which are then arranged in the form

$\begin{array}{lllllll}k l o & \text { ino } & j m o & i l m & j l n & i j k & k m n \\ i a b & j a c & l a d & n a e & k a f & m a g & o a h \\ n c d & m d b & k b c & \text { ocg } & m c h & l c e & i c f \\ m e f & k e g & i e h & j f b & \text { obe } & \text { ofd } & j d e \\ j g h & l h f & n f g & k h d & i d g & n h b & l b g\end{array}$

an arrangement, which, it may be remarked, contains eight different systems (such as have been considered in the former part of this paper) of seven letters such as $i, j$, $k, l, m, n, o$; and seven of other seven letters, such as $i, j, k, a, b, c, f\left({ }^{2}\right)$. The theory of the arrangement seems to be worth further investigation.

Assuming that the four hundred and fifty-five triads of fifteen things can be arranged in thirteen systems of thirty-five triads, each system of thirty-five triads containing every possible duad, it seems natural to inquire whether the thirteen systems can be obtained from any one of them by cyclical permutations of thirteen letters. This is, I think, impossible. For let the cyclical permutation be of the letters $a, b, c, d, e, f, g, h, i, j, k, l, m$. Consider separately the triads which contain the letter $n$ and the letter 0 ; neither of these systems of triads contains the letter, whatever it is, which forms a triad with $n$ and $o$. Hence, omitting the letters $n, o$, we have two different sets, each of them of six duads, and composed of the same twelve letters. And each of these systems of duads ought, by the cyclical permutation

1 The problem was proposed by Mr Kirkman, and has, to my knowledge, excited some attention in the form "To make a school of fifteen young ladies walk together in threes every day for a week so that each two may walk together." It will be seen from the text that I am uncertain as to the existence of a solution to the further problem suggested by Mr Sylvester, "to make the school walk every week in the quarter so that each three may walk together."

${ }^{2}$ [I have somewhat altered this sentence so as to express more clearly what appeared to be the meaning of it.]

$61-2$ 
in question, to produce the whole system of the seventy-eight duads of the thirteen letters. Hence arranging the duads of the thirteen letters in the form

$$
\begin{aligned}
& a b \cdot b c \cdot c d \cdot d e \cdot e f \cdot f g \cdot g h \cdot h i \cdot i j \cdot j k \cdot k l \cdot l m \cdot m a \\
& a c \cdot b d \cdot c e \cdot d f \cdot e g \cdot f h \cdot g i \cdot h j \cdot i k \cdot j l \cdot k m \cdot l a \cdot m b \\
& a d \cdot b e \cdot c f \cdot d g \cdot e h \cdot f i \cdot g j \cdot h k \cdot i l \cdot j m \cdot k a \cdot l b \cdot m c \\
& a e \cdot b f \cdot c g \cdot d h \cdot e i \cdot f j \cdot g k \cdot h l \cdot i m \cdot j a \cdot k b \cdot l c \cdot m d \\
& a f \cdot b g \cdot c h \cdot d i \cdot e j \cdot f k \cdot g l \cdot h m \cdot i a \cdot j b \cdot k c \cdot l d \cdot m e \\
& a g \cdot b h \cdot c i \cdot d j \cdot e k \cdot f l \cdot g m \cdot h a \cdot i b \cdot j c \cdot k d \cdot l e \cdot m f
\end{aligned}
$$

and consequently the duads of each set ought to be situated one duad in each line. Suppose the sets of duads are composed of the letters $a, b, c, d, e, f, g, h, i, j, k, l$, it does not appear that there is any set of six duads composed of these letters, and situated one duad in each line, other than the single set $a l, b k, c j, d i, e h, f g$; and the same being the case for any twelve letters out of the thirteen, the derivation of the thirteen systems of thirty-five triads by means of the cyclical permutations of thirteen letters is impossible. And there does not seem to be any obvious rule for the derivation of the thirteen systems from any one of them, or any prima facie reason for believing that the thirteen systems do really exist, it having been already shown that such systems do not exist in the case of seven things. 